\title{
图HAD
}

ISSN-L: 2530-5115

DOI: http://doi.org/10.22585/hospdomic.v1i4.30

\section{La importancia y necesidad del Digital Object Identifier (DOI)}

\section{The Importance and necessity of the Digital Object Identifier (DOI)}

\author{
Equipo editorial de la revista Hospital a Domicilio \\ Alicante, España.
}

\section{Correspondencia/Correspondence \\ editor@revistahad.eu}

Conflicto de Intereses/Competing interest:

No existe ningún tipo de conflicto de interés.

Financiación/Fundings:

No se declara ninguna fuente específica de financiación ni ayuda económica recibida.

\section{CÓMO CITAR ESTE TRABAJO | HOW TO CITE THIS PAPER}

Equipo editorial. La importancia y necesidad del Digital Object Identifier (DOI). Hosp Domic. 2017; 1(4): $185-7$

Digital Object Identifier (DOI) es, hoy en día, el indicador más usado para identificar cualquier entidad digital. Es una referencia en línea (digital) que apunta a la tipificación de un recurso (objeto). Esto permite que los DOI proporcionen una referencia estable a los usuarios de un objeto incluso si la dirección web (URL - Uniform Resource Locator) donde esté almacenado el recurso haya cambiado. Es un sistema parecido a los identificadores URI (Uniform Resource Identifier), cadena de caracteres que identifica los recursos de una red de forma unívoca.

DOI es un código suficientemente implantado y aplicable a cualquier tipo de objeto (artículo, libro, capítulo de libro, imagen, documento sonoro, producto audiovisual, etc.). Su principal aportación es asegurar la identificación persistente y univoca a un documento, a través de un registro sistemático de sus metadatos en un registro central (1).

DOI es, hoy en día, el indicador más usado para identificar cualquier entidad digital. Es una referencia en línea (digital) que apunta a la tipificación de un recurso (objeto). Esto permite que los DOI proporcionen una 
referencia estable a los usuarios de un objeto incluso si la dirección web (URL - Uniform Resource Locator) donde esté almacenado el recurso haya cambiado. Es un sistema parecido a los identificadores URI (Uniform Resource Identifier), cadena de caracteres que identifica los recursos de una red de forma unívoca.

Por tanto, a diferencia del sistema URL, usado para localizar las páginas web, el sistema DOI no cambia con el paso del tiempo, aunque el objeto (documento) sea reubicado en una dirección distinta puesto que lleva la información incorporada en forma de metadatos.

El DOI consiste en un código alfanumérico que identifica en la web un artículo y que lo recupera incluso si éste se ubica en un servidor distinto al que fue alojado en un principio, evitando así el típico problema de los enlaces rotos (2).

El sistema DOI se construye a partir de varios estándares existentes, basados en componentes que hacen que el código final sea único e irrepetible. El sistema ha sido aceptado para su estandarización por la Organización Internacional de Estandarización (ISO) con el registro ISO 26324:2012. EI DOI está constituido por dos componentes: el prefijo y el sufijo. Juntos forman el identificador. Los dígitos colocados después de la diagonal (barra inclinada), el sufijo del DOI, puede corresponder a un identificador existente, mientras que los ubicados antes de la diagonal, el prefijo del DOI, pertenecen a un nombre único de autoridad. En la extensión del DOI no hay una limitación (2).

El sistema está gestionado por la International DOI Foundation (https://www.doi.org/), aunque el registro se realiza a través de Agencias de Registro. En el caso de la revista Hospital a Domicilio, a través de Crossref (https://www.crossref.org/). Crossref es un consorcio de editores sin ánimo de lucro, al que se ha adherido la revista Hospital a Domicilio, que realiza los servicios de registro para las entidades miembro.

La estructura del identificador DOI se compone de dos partes, separadas por una barra inclinada: Prefijo/Sufijo.

- Prefijo, asignado al editor (editorial) por una agencia registradora.

- Sufijo, designación dada por el editor (editorial) al contenido específico que se va a identificar.

Por ejemplo, la estructura DOI de artículo, 10.22525/hospdomic.v1i1.6, publicado en la revista Hospital a Domicilio, puede leerse de la manera que se muestra en la tabla 1.

\begin{tabular}{|c|c|c|c|}
\hline \multicolumn{2}{|c|}{ Prefijo } & \multicolumn{2}{|c|}{ Sufijo } \\
\hline $\begin{array}{l}\text { Código de la agencia } \\
\text { registradora del DOI }\end{array}$ & $\begin{array}{l}\text { Código del editor } \\
\text { que asigna el sufijo }\end{array}$ & $\begin{array}{l}\text { Código de la revista } \\
\text { (asignado por el editor) }\end{array}$ & $\begin{array}{l}\text { Código del artículo } \\
\text { (asignado por el editor) }\end{array}$ \\
\hline 10 & 22585 & hospdomic & v1i1.6 \\
\hline
\end{tabular}

$\mathrm{Si}$ al indicador anterior se le agrega la dirección http://dx.doi.org/, es decir http://dx.doi. org/10.22525/hospdomic.v1i1.6, se dirige a la base de datos del DOI y de allí el sistema enlaza automáticamente con el documento (objeto digital), permitiendo el rápido enlace con él.

Por tanto, mediante el DOI, cualquier artículo publicado en nuestra revista puede ser fácilmente rastreable y su contenido es susceptible de búsqueda por las herramientas disponibles (3). 
Desde el primer número de la revista Hospital a Domicilio, ya se tuvo en cuenta la asignación a cada uno de los artículos del DOI, convencidos de que era un compromiso editorial para preservar los artículos que se publicaran y conscientes de que las referencias incluidas en la bibliografía de un artículo serían, en muchas ocasiones, consultadas y estudiadas (4). Y, permite hacer efectivo el compromiso de mantener la disponibilidad de cualquier artículo publicado.

El código DOI no es la única forma de obtener identificaciones fijas, pero se ha convertido en el sistema principal. Debido a esta fortaleza, si un artículo científico cuenta con correspondiente código DOI, se puede tener la garantía de que podrá ser detectable y que su referencia no desaparecerá.

\section{BIBLIOGRAFÍA}

1. Rodríguez-Yunta L. Pensando en digital: uso del doi en revistas españolas, una asignatura aún pendiente. Anu ThinkEPI. 2013;7:164-8.

2. El editor. En síntesis: El DOI, Identificador de Objetos Digitales. Inf Tecnológica. 2011;22(6):1. DOI: $10.4067 /$ S0718-07642011000600001

3. Goldenberg D. The importance of the DOI Digital Object Identifier. Rev Bras Cir Plástica. 2014;29(3):311. DOI: 10.5935/2177-1235.2014RBCP0058

4. Sanz-Valero J, Wanden-Berghe C. Análisis bibliométrico de la producción científica, indizada en MEDLINE, sobre los servicios de salud proporcionados por las unidades de hospitalización a domicilio. Hosp Domic. 2017;1(1):21. DOI: 10.22585/hospdomic.v1i1.3 